\title{
Structural conditioning factors of the installation of rooftop hvac equipment on existing load-bearing masonry buildings: Case study Condicionantes estructuralles de la implantación de instalaciones de climatización en cubiertas de edificios existentes de muros de carga: Caso de estudio
}

T. Martins ${ }^{1 *}$, J. Fernández **, J. Fernández *

* Universidad Politécnica de Madrid - Madrid, ESPAÑA

** Universidad Politécnica de Valencia - Valencia, ESPAÑA

Fecha de Recepción: 24/02/2020

Fecha de Aceptación: 18/02/2021

PAG 88-96

\begin{abstract}
The growing interest in providing adequate Heating, Ventilation and Air Conditioning (HVAC) conditions for those public buildings in which they were not originally planned frequently leads to the need to install HVAC units of significant dimensions and weights in these buildings. Particularly in buildings located in urban centers, the unavailability of space to place those units has led to their installation in buildings' roofs.

From the case study of a remarkable brick masonry building located in the historical city center of Madrid, this article analyzes the structural aspects involved in the implementation of large-size HVAC units on the roof of existing masonry buildings, as well as the structural refurbishment typologies necessary for this purpose.

Thus, the study carried out focused, on the one hand, on the analysis of the HVAC new loads' impact on the previous masonry safety conditions, and the possible need to adopt strengthening actions to ensure an adequate future structural behavior, and, on the other hand, it focuses on the analysis of the aspects conditioning the design of the auxiliary support and fixing structure of those HVAC units.
\end{abstract}

Keywords: Brick masonry, structural strengthening, HVAC units, change in use, structural assessment

Resumen

El interés creciente por dotar de unas adecuadas condiciones de climatización a los edificios de uso público que no disponían en origen de éstas conlleva, en muchos casos, a la necesidad de implantar instalaciones de climatización de dimensiones y pesos significativos. Máxime en el caso de edificios situados en núcleos urbanos, la ausencia de superficie disponible para implantar dichas instalaciones ha motivado que se dispongan en sus cubiertas.

A partir del caso de estudio de un edificio notable de fábrica de ladrillo del casco histórico de Madrid, se analizan los condicionantes estructurales que supone la implantación de importantes aparatos de climatización en la cubierta de edificios existentes de muros de carga, así como las tipologías de actuación de acondicionamiento estructural necesarias al efecto.

Asimismo, el estudio realizado se centró, por una parte, en el análisis de la repercusión de la disposición de nuevas cargas sobre las condiciones de seguridad previas de la estructura y la necesidad de adoptar en ésta medidas de refuerzo que permitan asegurar unas adecuadas condiciones de seguridad futuras y, por otro lado, en el análisis de los condicionantes a tener en cuenta en el diseño de la estructura auxiliar de apoyo y sujeción de dichas instalaciones.

Palabras clave: Fábrica de ladrillo, refuerzo estructural, instalaciones de climatización, cambio de uso, evaluación estructural

\section{Introduction}

Entre las principales razones para intervenir en edificios históricos se encuentra la necesidad de modernizar y optimizar sus sistemas de climatización, de cara a mejorar su eficiencia energética y/o responder a las demandas de confort térmico requeridas actualmente para sus usuarios (Park, 1991) (Pérez-Lombard et al., 2008) (Ma Z et al., 2012) (Pisello et al., 2014). En el caso de los edificios situados en núcleos urbanos, que no disponían en origen de unas adecuadas condiciones de climatización, la ausencia de superficie disponible para implantar las instalaciones necesarias a su mejora ha motivado que en muchos casos éstas se dispongan en la cubierta de los mismos, bien apoyadas en subestructuras colocadas al efecto sobre la superficie de la cubierta, o bien directamente sobre la estructura portante de los inmuebles (AHRI, 1997).Además, en los edificios de uso público, por su volumetría, se requieren en general aparatos de climatización de dimensiones y pesos significativos que permitan dar respuesta a dichas exigencias de mejora de sus condiciones de climatización (Alderson, 2009) (Westphalen y Koszalinski, 2001) (Westphalen y Koszalinski, 1999). Esto conlleva, habitualmente, a la necesidad de tener que realizar un análisis previo de la repercusión sobre las condiciones de seguridad nominales de las estructuras a intervenir frente a las nuevas cargas a disponer-, que permita determinar si resulta necesario adoptar alguna medida de refuerzo estructural de cara a asegurar unas adecuadas condiciones de seguridad y servicio futuras de los edificios tras la actuación. Por los mismos motivos, suele resultar igualmente preciso la realización de un estudio estructural específico que permita determinar los condicionantes que imponen los edificios a intervenir al diseño y situación de las subestructuras de apoyo y sujeción de dichas instalaciones.

\footnotetext{
1 Corresponding author:

Universidad Politécnica de Madrid - Madrid, ESPAÑA

E-mail: tiago.martins@upm.es
} 
Cabe señalar, no obstante, que en la bibliografía de referencia la investigación sobre la disposición de instalaciones de climatización en edificios históricos se viene centrando fundamentalmente en aquellos aspectos que condicionan el ahorro o eficiencia energética o la calidad del aire interior que se alcanzan con los aparatos a disponer (Webb, 2017) (Cabeza et al, 2018) (Krzemień et al., 2018). El estudio realizado tiene, en este sentido, por objetivo poner de relieve la importancia de los condicionantes estructurales en la toma de decisiones sobre la elección de dichas instalaciones habida cuenta, además, de que se trata de aparatos que, en general, presentan cargas importantes y que se disponen en la cubierta de edificios antiguos.

A partir de un caso de estudio, se aborda en el presente artículo esta problemática aplicada a los edificios de fábrica, analizando tanto los condicionantes estructurales que supone la implantación de importantes instalaciones de climatización en la cubierta de edificios antiguos de muros de carga, como las soluciones de acondicionamiento estructural necesarias al efecto.

El inmueble objeto de análisis se construyó en el siglo XVIII. Se trata de un notable edificio de uso público situado en el casco histórico de Madrid y, cuya estructura está formada por forjados unidireccionales constituidos por viguetas metálicas que apoyan sobre muros de fábrica de ladrillo (Figura 1). Sobre este edificio se ha decidido llevar a cabo una actuación generalizada de mejora de sus sistemas de climatización que obligó a disponer diferentes instalaciones en la cubierta del mismo: unidades de tratamiento de aire, enfriadoras, bombas de calor, equipos autónomos, etc.

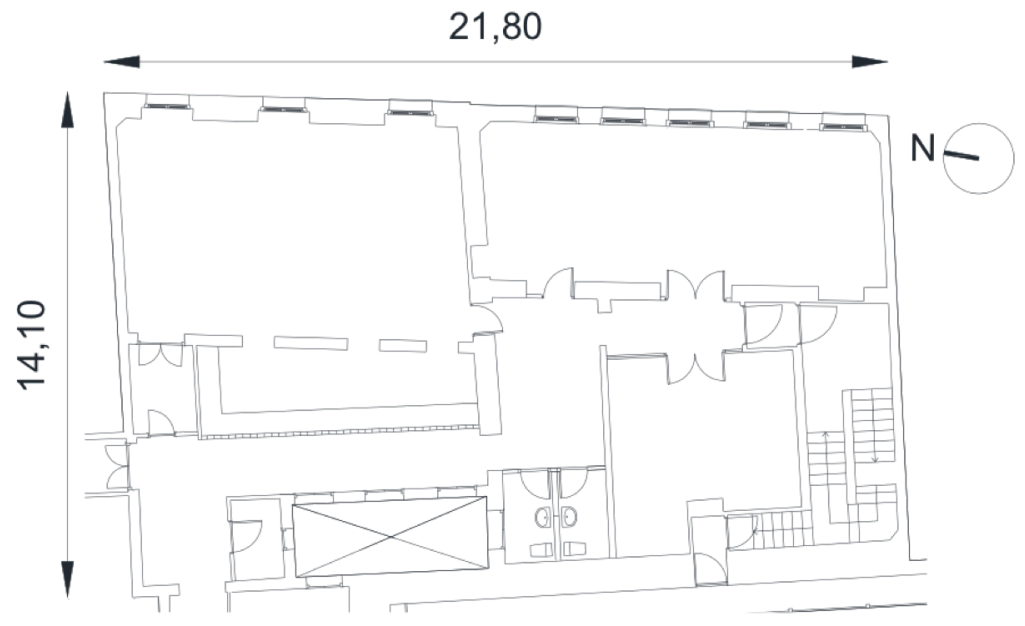

Figura 1. Croquis parcial de la planta bajo cubierta del inmueble objeto de análisis, correspondiente a la zona del edificio sobre la que se pretenden disponer dos unidades de tratamiento de aire

\section{Ingestigaciones previas}

\subsection{Trabajos de inspección}

Se realizó primeramente una inspección del edificio con el objeto de averiguar la existencia en éste de daños o anomalías indicativos de un anómalo comportamiento estructural. De acuerdo con los resultados obtenidos en dichos trabajos de inspección, el inmueble no presentaba indicios de un anómalo comportamiento de su estructura.

\subsection{Campaña de calas en estructura}

Tras la inspección de daños se llevó a cabo una campaña de calas en la cubierta (Figura 2) y en algunos tramos de muros de carga bajo la misma con el objeto determinar su configuración. 


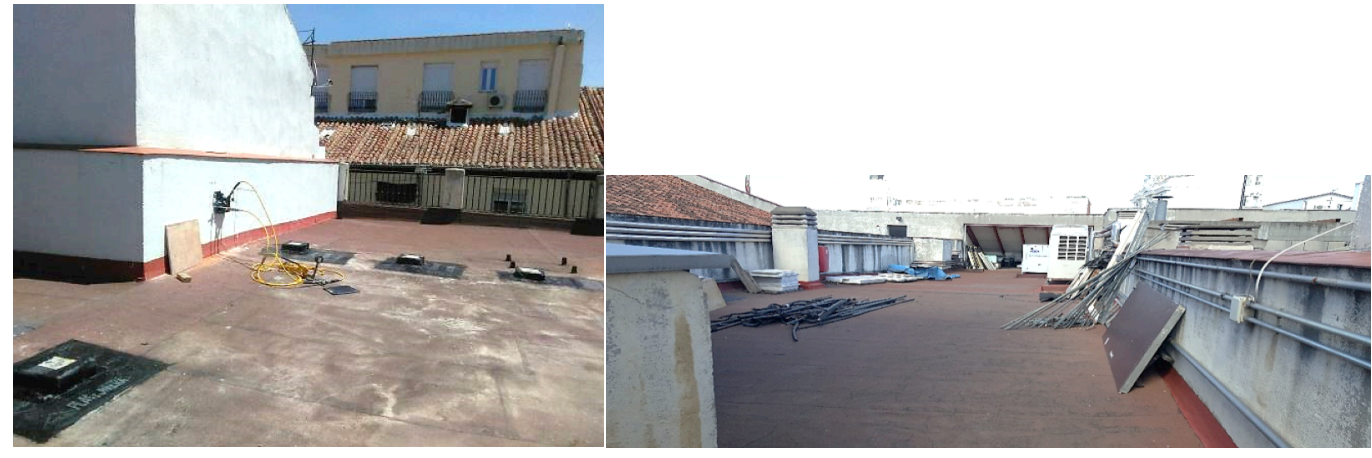

Figura 2. Vistas generales de la cubierta del edificio

Los aspectos más significativos recabados en estos trabajos de investigación fueron los siguientes:

- El revestimiento de la estructura de cubierta se resuelve con tabiques palomeros de ladrillo de $20 \mathrm{~cm}$ de altura sobre los que se disponen diferentes capas de remate de la cubierta (rasillas, laminas impermeabilizantes, etc.) hasta configurar una altura total del conjunto de unos $30 \mathrm{~cm}$ (Figura 3).

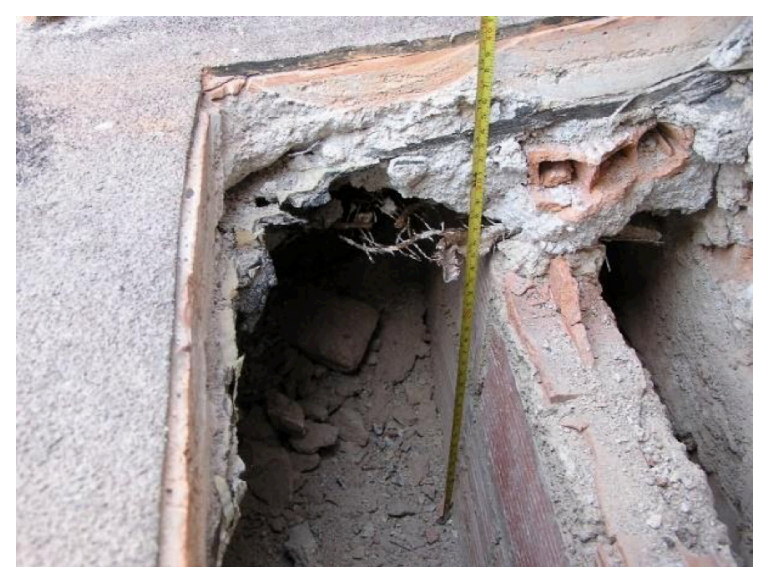

Figura 3. Vista de una de las calas realizadas en la cubierta del edificio

- Los forjados de la cubierta son unidireccionales, formados por viguetas metálicas tipo IPN 160, con un intereje de $75 \mathrm{~cm}$ y entrevigado de revoltón de fábrica de una rosca de ladrillo y una capa de reparto de entre $2 \mathrm{~cm}$ y $3 \mathrm{~cm}$.

- Los muros de carga son de ladrillo y presentan espesores de entre un pie y dos pies.

\subsection{Análisis de repercusión estructural de las nuevas cargas a disponer en cubierta}

Con la información obtenida en la campaña de calas en estructura y la que se recogía en la documentación técnica disponible sobre el inmueble, se llevaron a cabo comprobaciones de seguridad sobre los elementos con misión resistente que se verían sobresolicitados tras la intervención prevista, de cara a determinar las medidas de acondicionamiento estructural que pudiera resultar necesario acometer en el edificio con el objeto de asegurar unas adecuadas condiciones de seguridad futuras del mismo tras la actuación.

Cabe señalar que, teniendo en cuenta que se trataba de una cubierta cuyo revestimiento estaba conformada por tabiques palomeros, sobre los que no se podían apoyar directamente las cargas a disponer, se planteó como solución para el apoyo de las instalaciones a disponer en cubierta, referir de forma directa las cargas de los aparatos a los tramos de muros de carga existentes. Así pues, no resultó, en este caso, necesaria la comprobación de la estructura horizontal de cubierta frente a dichas cargas.

En relación con los tramos de muro afectados por la intervención prevista, se llevó a cabo un análisis tensional de los mismos frente a las nuevas cargas a disponer en cubierta. No obstante, en dicho análisis no se 
dedujo la necesidad de acometer medidas de refuerzo en los tramos de muro solicitados por dichas cargas. Esto se debió a que la superficie que quedaba ocupada por los emparrillados metálicos de apoyo de los aparatos a disponer era importante, dado que resultaba necesario llevar sus apoyos hasta la posición de las fábricas. De este modo, los nuevos estados tensionales en los tramos de muro afectados resultaron ser análogos a los anteriores debidos a la sobrecarga de uso en cubierta correspondiente a esas superficies.

\section{Planteamiento de la solución de apoyo de instalaciones}

Como se ha indicado, en diferentes recuadros de la cubierta objeto de análisis, se necesitaba disponer diversas instalaciones de climatización: varias unidades de tratamiento de aire (UTA), enfriadoras, bombas de calor, equipos autónomos, etc. Cabe indicar que, teniendo en cuenta que la solución elegida para la disposición de las instalaciones fue la de referir de forma directa las nuevas cargas a los muros existentes mediante emparrillados metálicos diseñados al efecto, la geometría y configuración de éstos quedó condicionada por la distribución de la estructura de fábrica, por la situación de los apoyos de los aparatos a disponer y además por las alineaciones de una estructura metálica que se había dispuesto con anterioridad en la cubierta.

Asimismo, se plantearon cuatro estructuras metálicas, a modo de emparrillados, constituidas por perfiles de acero laminado de tipo IPE 180, IPE,160, IPE 140 y HEB 140, sobreelevadas respecto de la cubierta, y apoyadas, bien sobre soportes de reducida longitud-conformados por perfiles tubulares de $100 \mathrm{~mm} \times 100 \mathrm{~mm} \times 5 \mathrm{~mm}-$, que descansan a su vez sobre las fábricas existentes bajo la cubierta, o bien ancladas mediante placas y varillas a los tramos de muro que quedaban por encima de la cota del emparrillado (muros sobreelevados, petos, etc.). Se trató igualmente de que las alineaciones en planta de los emparrillados a diseñar fuesen aproximadamente ortogonales entre perfiles y que las uniones entre estos elementos fuesen soldadas. Además, se planteó disponer, en el perímetro de los aparatos de climatización, plataformas con rejilla metálica tipo tramex para la conservación y mantenimiento de los mismos. Como opciones de enlace de las vigas a los muros, se plantearon dos soluciones: una con soldadura de la viga a placa de anclaje y otra con pasadores a modo de liberar a los muros de posibles empujes horizontales.

Así pues, teniendo en cuenta los condicionantes existentes para el diseño de las soluciones de apoyo de los aparatos de climatización antes indicados, se plantearon las tipologías de emparrillado que se indican a continuación:

Emparrillado 1: Se plantea un único emparrillado metálico para el apoyo de enfriadoras, bomba de calor y equipos autónomos, conformado por perfiles tipo IPE 180, IPE 160 y IPE 140, aprovechando para su apoyo los soportes de una estructura metálica dispuesta en la cubierta con anterioridad -dando continuidad a sus alineaciones- y creando cinco puntos de apoyo adicionales en los tramos de muro existentes bajo dicho emparrillado (Figura 4). Para ello, se planteó perforar puntualmente el forjado para realizar macizados hasta alcanzar los tramos de muro bajo cubierta donde apoyar el emparrillado. El resto de los puntos de apoyo se materializan mediante placas de anclaje a las fábricas cuya cota superior queda por encima del emparrillado. Cabe indicar que en el extremo de esta estructura se crea un voladizo a partir de los nuevos soportes (puntos de apoyo) sobre los tramos de muro bajo cubierta.

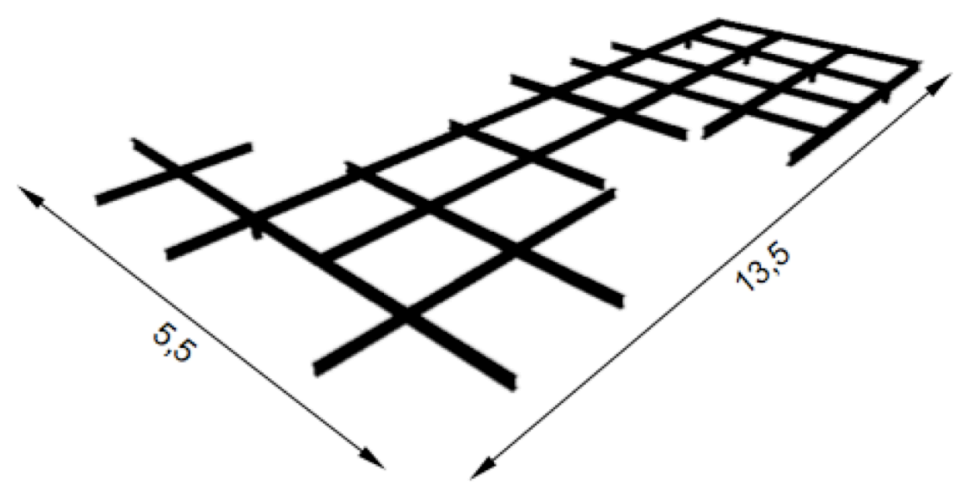

Figura 4. Representación gráfica de la configuración del Emparrillado 1 
Emparrillado 2: Constituido por tres perfiles tipo IPE 160, con apoyos en placas referidas a los muros sobreelevados, y por perfiles tipo IPE 140 transversales (Figura 5), y sobre los que descansará en servicio la plataforma y los apoyos de una unidad de tratamiento de aire.

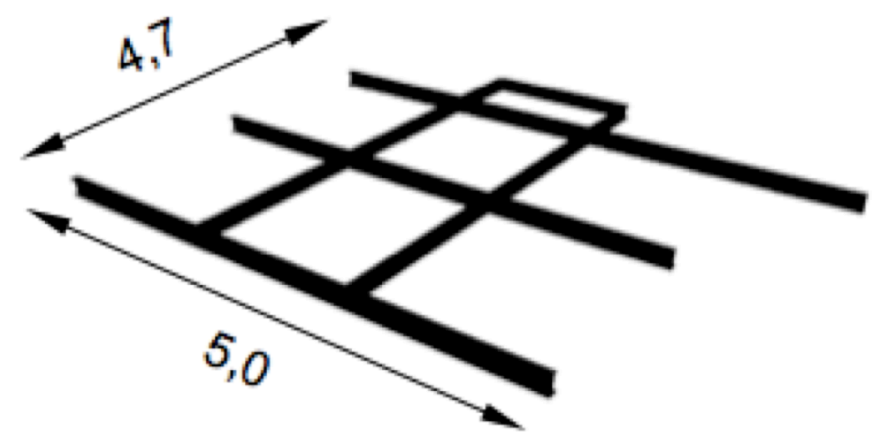

Figura 5. Representación gráfica de la configuración del Emparrillado 2

Emparrillado 3: Se materializa con dos perfiles tipo IPE 160, referidos a los tramos de muro sobreelevados mediante placas de anclaje, y dos perfiles transversales tipo IPE 140 (Figura 6), y sobre los que descansará en servicio la plataforma y los apoyos de otra UTA y de las bombas de calor.

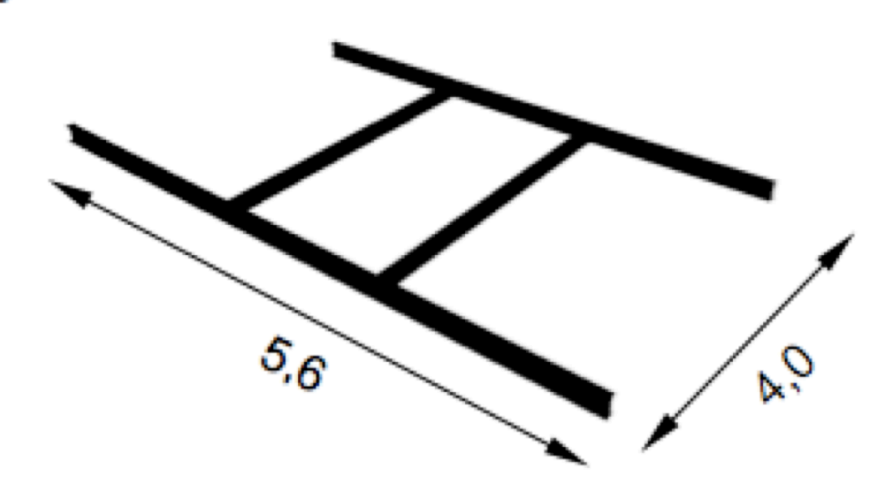

Figura 6. Representación gráfica de la configuración del Emparrillado 3

Emparrillado 4: Constituido por perfiles de tipo IPE 160 e IPE 140. Sirve para el apoyo de dos UTA. Está igualmente sobreelevado respecto de la cubierta y apoyado, bien sobre soportes de reducida longitud, que descansan a su vez sobre los muros portantes de fábrica existentes bajo la cubierta, o bien anclado mediante placas y varillas a los muros perimetrales de fábrica que quedan por encima de la cota del emparrillado (Figura 7). Éste cuenta, además, con un perfil de tipo HEB 140, que descansa sobre la cabeza de un muro ligeramente sobreelevado de la cubierta -anclado al mismo mediante placas y varillas-, y que coincide con el apoyo medianero de las dos UTA a disponer sobre esta estructura. Cabe señalar que, dada la configuración de los muros bajo este emparrillado, es necesario, también en este caso, crear en el extremo noreste del mismo un voladizo a partir de los puntos de apoyo sobre los muros de los nuevos soportes y del perfil tipo HEB 140. 


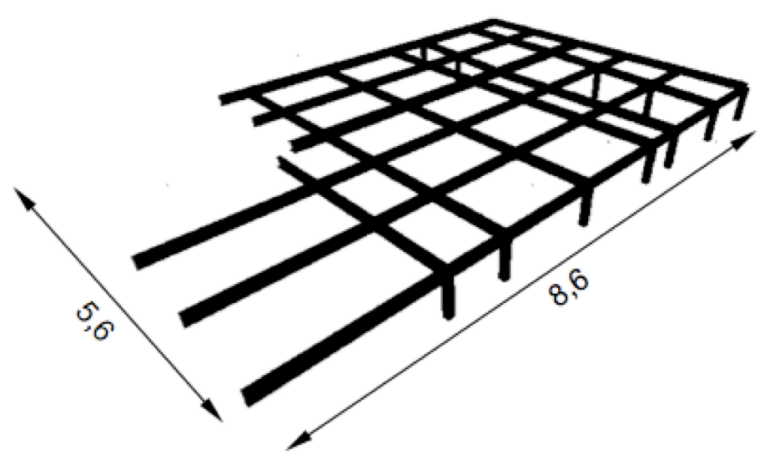

Figura 7. Representación gráfica de la configuración del Emparrillado 4

\section{Comprobaciones de cálculo}

A partir de las soluciones, que se describen en el apartado anterior, planteadas para las cuatro estructuras metálicas de apoyo de los diferentes aparatos de climatización a instalar en cubierta, se llevaron a cabo las comprobaciones de cálculo necesarias para su diseño.

\subsection{Diseño de emparrillados}

Se realizó mediante el empleo de la herramienta informática de cálculo estructural METAL $3 \mathrm{D}^{\circledR}$ de CYPE INGENIEROS (Figura 8), comprobando que los perfiles planteados en el prediseño de los emparrillados (véase el apartado anterior) eran acordes con las necesidades de dimensionamiento frente a las acciones previstas sobre los mismos. Se verificó, asimismo, en cuanto a las deformaciones en perfiles, que las flechas relativas obtenidas eran inferiores a 1/250 de la luz en todos los casos.

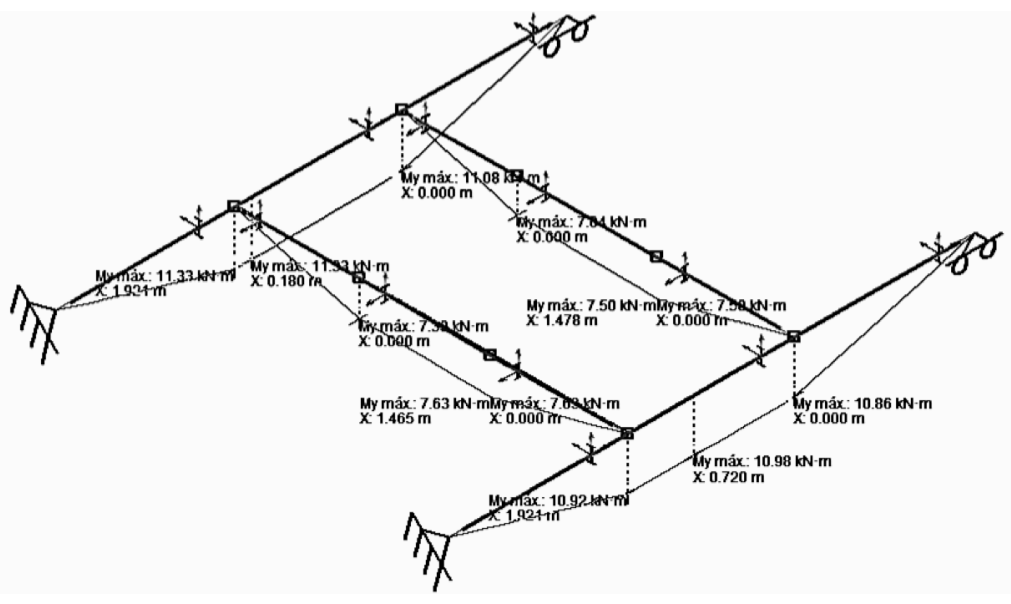

Figura 8. Leyes de momentos flectores del Emparrillado 3 


\subsection{Diseño de anclajes}

El diseño de los anclajes de los emparrillados a los muros de fábrica sobreelevados del inmueble se realizó de acuerdo con los criterios de diseño y comprobación de anclajes que se establecen en la guía técnica de anclajes de $\mathrm{HILTI}^{\circledR}(\mathrm{HILTI}, 2019)$. Asimismo, teniendo en cuenta las soluciones válidas para las acciones obtenidas en los resultados del cálculo estructural previamente realizado, y adoptando unas características resistentes conservadoras para los muros de fábrica existentes en el edificio, a expensas de la realización de ensayos de verificación posteriores, resultaba necesario disponer varillas de anclaje Ø12 HIT-V con anclaje químico HIT-HY 270.

No obstante, puesto que no se disponía de información detallada sobre las características resistentes del material de soporte de los anclajes, se estimó además oportuno la realización de ensayos in situ de tracción y corte -que se describen en el siguiente apartado-, al objeto de garantizar un adecuado comportamiento en servicio de los anclajes diseñados.

\section{Campaña de ensayos}

Previamente a la ejecución de los anclajes planteados para el apoyo y sujeción de los emparrillados, con el objeto de verificar la idoneidad de los mismos, se llevó a cabo una campaña de ensayos de tracción y corte (Figura 9) sobre unos anclajes de idéntica tipología que los diseñados para el emparrillado, de acuerdo con las prescripciones de ensayo que se recogen en (EOTA TR 053, 2016).
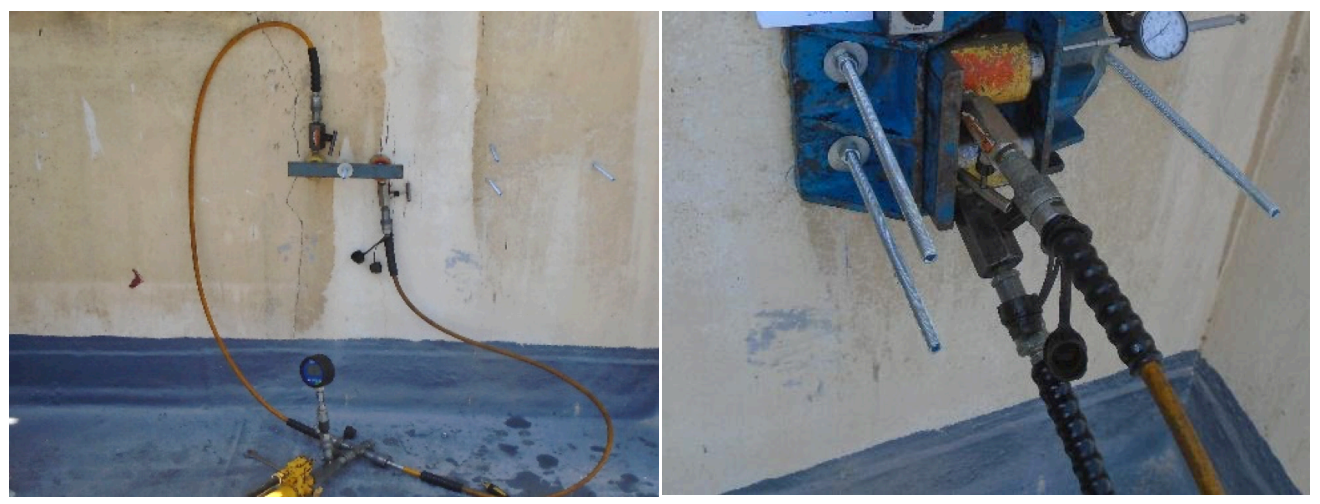

Figura 9. Ensayos de tracción y corte de anclajes

Se estableció como criterio de aceptación para los ensayos a realizar que estos serían válidos para valores de carga de tracción superiores a $3 \mathrm{kN}$ y para valores de corte superiores a $6 \mathrm{kN}$.

En la (Tabla 1) siguiente se presentan los resultados alcanzados en los ensayos realizados. Se recogen igualmente en la Figura 10 las gráficas de comportamiento carga-deformación obtenidas.

Tabla 1. Resultados obtenidos en los ensayos de corte y tracción de anclajes

\begin{tabular}{|c|c|}
\hline Ensayo & Carga de ensayo $(\mathbf{k N})$ \\
\hline E1 - Tracción & 4,11 \\
\hline E2 - Cortante & 9,55 \\
\hline E3 - Tracción & 6,43 \\
\hline E4 - Tracción & 3,16 \\
\hline E5 - Tracción & 4,06 \\
\hline E6 - Tracción & 4,06 \\
\hline E7 - Cortante & 9,40 \\
\hline E8 - Cortante & 9,10 \\
\hline
\end{tabular}



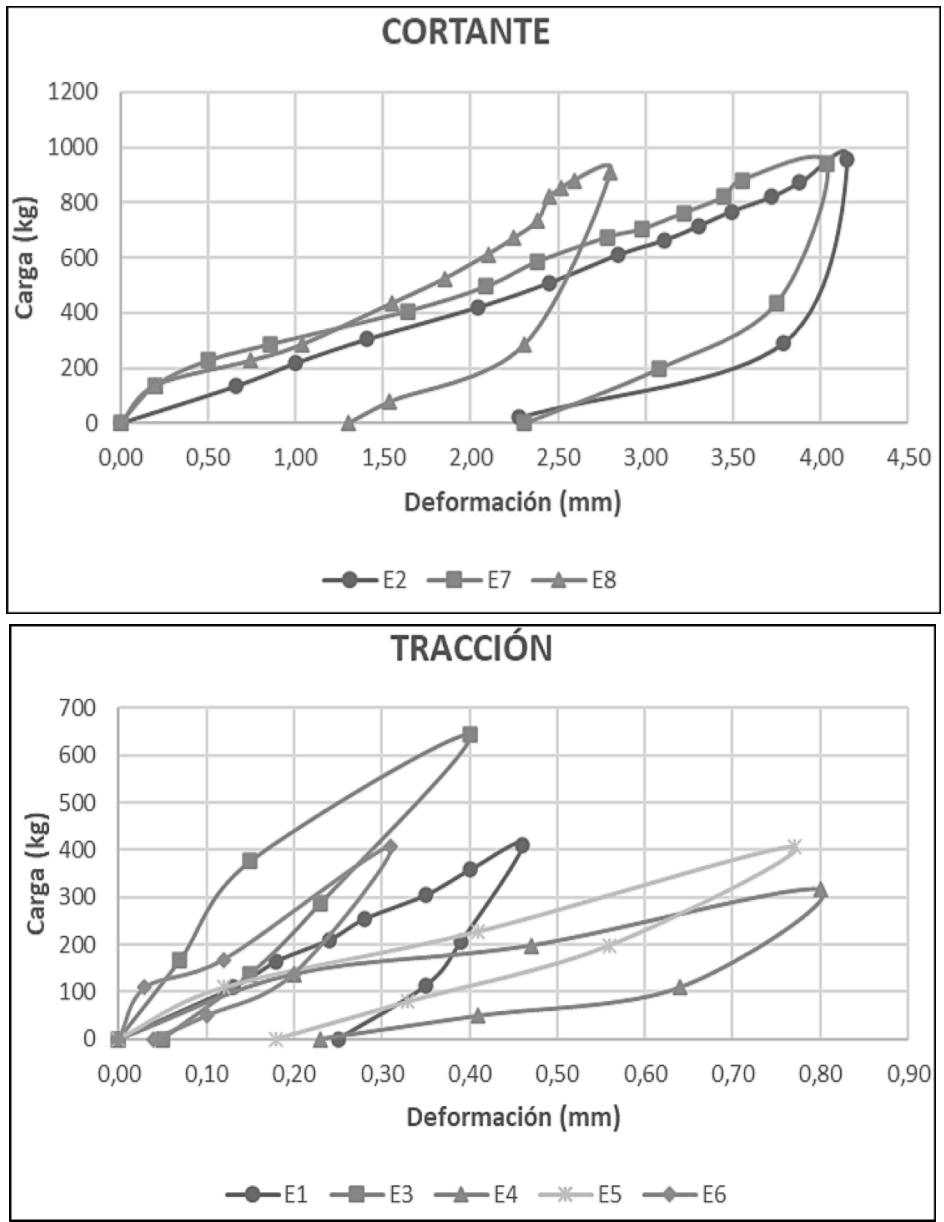

Figura 10. Curvas de comportamiento obtenidas en los ensayos de corte y de tracción

\section{Controll del montaje}

Tras las comprobaciones de diseño de las soluciones de apoyo y sujeción de los aparatos y la realización de los ensayos de verificación de la estructura existente, de cara a garantizar un adecuado comportamiento, tanto de las instalaciones a disponer como de las estructuras sobre las que van a descansar, resulta igualmente importante verificar in situ la adecuación del montaje de los aparatos a lo previamente definido (Figura 11).

Este control permite, asimismo, comprobar que éstos se disponen de forma adecuada y de acuerdo con los requisitos de calidad establecidos previamente para el mismo, máxime en lo relativo a la posición de sus apoyos, y que las características de los mismos se adecúan a sus prescripciones técnicas. Cabe señalar además que, en zonas urbanas densamente pobladas como la que es objeto de análisis, estas operaciones de montaje cuentan con la dificultad añadida de realizarse por la noche y mediante izado con grúa de los aparatos desde planta baja. 


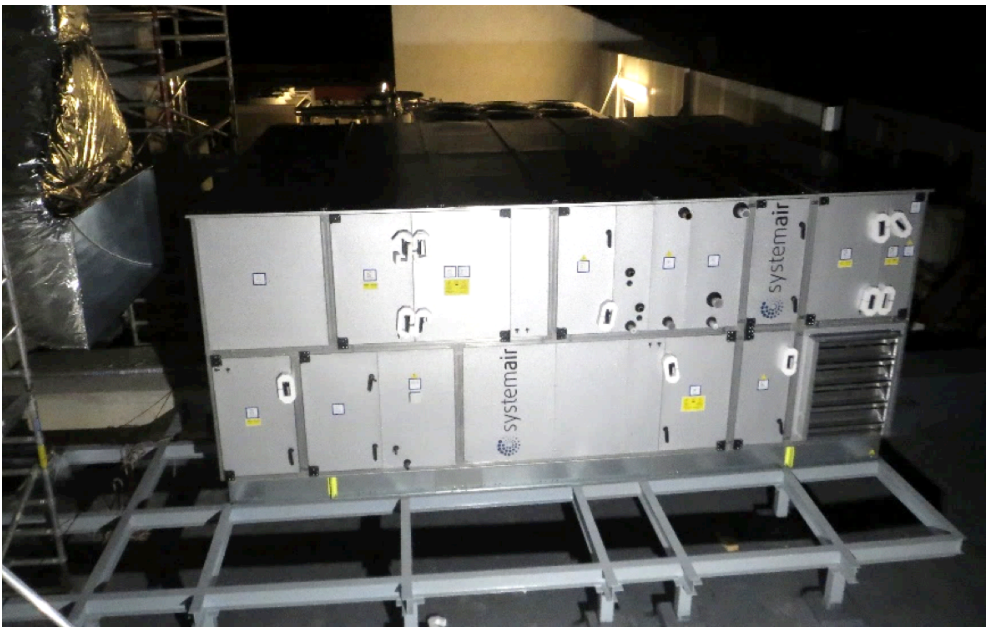

Figura 11. Disposición de dos UTA tras montaje

\section{Conclusiones}

El caso de estudio objeto de investigación en este artículo permitió poner de relieve la existencia de diferentes condicionantes que influyen, en mayor o menor medida, en la toma de decisiones sobre la viabilidad de disposición de importantes instalaciones de climatización en la cubierta de edificios antiguos de uso público, y que deben por ello ser objeto de comprobación, entre los que cabe destacar: (i) La configuración y tipología de la estructura existente; (ii) La existencia de daños indicativos de un anómalo comportamiento estructural del inmueble; (iii) La necesidad de realización de ensayos de caracterización de la estructura; (iv) La repercusión de las nuevas cargas a disponer sobre las condiciones de seguridad previas de la estructura; (v) La configuración y diseño de las estructuras auxiliares de apoyo de los aparatos a instalar; (vi) La adecuación del montaje.

El análisis de estos aspectos resulta, asimismo, determinante para el desarrollo de las soluciones de acondicionamiento estructural de estos edificios que permitan garantizar unas adecuadas condiciones de seguridad y servicio tanto de su estructura como de las instalaciones a disponer en cubierta.

\section{Referencias}

AHRI (1997). Air-Conditioning, Heating, and Refrigeration Institute: Guidelines for Roof Mounted Outdoor Air-Conditioner Installations.

Alderson C., (2009). HVAC Upgrades in Historic Buildings. Technical Preservation Guidelines.

Cabeza, L. F.; De Gracia, A.; Pisello, A. L. (2018). Integration of renewable technologies in historical and heritage buildings: A review. Energy and Buildings, 177, 96-111. doi:10.1016/j.enbuild.2018.07.058

EOTA TR 053 (2016). European Organisation for Technical Approvals: TR 053: Recommendations for Job-site Tests of Metal Injection Anchors for Use in Masonry.

HILTI (2019). North American Product Technical Guide, Volume 2: Anchor Fastening Technical Guide, Edition.

Krzemień, L.; Kupczak, A.; Pretzel, B.; Strojecki, M.; Radoń, J.; Bogaczewicz-Biernacka, E. (2018). Different HVAC systems in historical buildings to meet collection demands. In Conference Report: The 3rd International Conference on Energy Efficiency in Historic Buildings (pp. 337-344).

Ma, Z.; Cooper, P.; Daly D.; Ledo L. (2012). Existing building retrofits: Methodology and state of the art. Energy and Buildings, 55, 889902. https://doi.org/10.1016/j.enbuild.2012.08.018.

Park, SC. (1991). Heating, Ventilating, and Cooling Historic Buildings: Problems and Recommended Approaches. Preservation Brief, 24.

Pérez-Lombard L.; Ortiz J.; Pout C. (2008). A review on buildings energy consumption information. Energy and Buildings, 40(3), 394398. https://doi.org/10.1016/j.enbuild.2007.03.007.

Pisello A. L.; Petrozzi A.; Castaldo V. L., Cotana F. (2014). Energy Refurbishment of Historical Buildings with Public Function: Pilot Case Study. Energy Procedia, 61, 660-663. https://doi.org/10.1016/j.egypro.2014.11.937.

Webb, A. L. (2017). Energy retrofits in historic and traditional buildings: A review of problems and methods. Renewable and Sustainable Energy Reviews, 77, 748-759. doi:10.1016/j.rser.2017.01.145

Westphalen D.; Koszalinski S. (1999). Energy Consumption Characteristics of Commercial Building HVAC Systems Volume II: Thermal Distribution, Auxiliary Equipment, and Ventilation.

Westphalen D.; Koszalinski S. (2001). Energy Consumption Characteristics of Commercial Building HVAC Systems Volume I: Chillers, Refrigerant Compressors, and Heating Systems. 